\title{
Toxoplasma infection and systemic lupus erythematosus: Analysis of the serological response by immunoblotting
}

\author{
I Noel, A H Balfour, $\mathrm{M} \mathrm{H}$ Wilcox
}

\begin{abstract}
Aims-To examine the serological response of patients with systemic lupus erythematosus (SLE) and toxoplasma infection and to compare the blot profiles with those from immunocompetent subjects of similar immune response.

Methods-Forty serum samples from patients with SLE were tested for toxoplasma antibodies using the dye and indirect haemagglutination tests. Specific IgM was measured by $\mu$-capture enzyme linked immunosorbent assay (ELISA). The sera were immunoblotted using antigen strips prepared from the RH strain of Toxoplasma gondii. For comparison, control blots were prepared from pooled sera from immunocompetent subjects with serological evidence of acute (pool 1), or chronic (pool 2) toxoplasma infection, or with no evidence of infection (pool 3).

Results-Some of the blot profiles from the patients with SLE were compatible with the corresponding serology but others showed considerable variation, particularly among the IgM blots. The blots from sera with low dye test titres suggested that the latter could be false positive results.

Conclusions-Toxoplasma infection may enhance the production of autoantibodies which, when combined with the high titres characteristic of SLE, might interfere in the dye test and other serological tests. Immunoblotting could prove useful in the immunocompromised for confirming the presence of specific toxoplasma antibodies and for the staging of infection in those with positive serology.
\end{abstract}

(f Clin Pathol 1993;46:628-632)

The Toxoplasma

Reference Laboratory,

Public Health

Laboratory, Leeds

I Noel

A H Balfour

Bacteriology

Department, Royal

Hallamshire Hospital,

Sheffield

M H Wilcox

Correspondence to:

Dr I Noel, Department of

Pathology, Dryburn

Hospital, North Road

Durham DH1 5TW

Accepted for publication

18 March 1993 the fetus. In the immunocompromised-for example, transplant recipients and those with AIDS - toxoplasmosis is an important cause of morbidity and mortality. ${ }^{34}$ Symptoms in these patients are frequently a consequence of reactivation of a chronic, previously latent, toxoplasma infection. ${ }^{5}$ The mainstay of diagnosis is still serology, in particular the Sabin Feldman dye test, ${ }^{6}$ but results may be difficult to interpret in those who are immunocompromised.

A report by Wilcox et al has illustrated the problem of diagnosing toxoplasmosis in a further group of immunocompromised patients-those with systemic lupus erythematosus (SLE). ${ }^{7}$ Clinical diagnosis of toxoplasmosis in the presence of SLE is difficult because the two diseases produce similar symptoms. The authors therefore undertook a serological assessment of a group of patients with SLE and found that high titres of toxoplasma antibody were significantly more common in these patients than in healthy controls. The titres did not correlate with any of the routinely measured indices in SLE nor with the patients' previous treatment. In our study immunoblotting was used to characterise the peptide patterns recognised by dye test positive and negative sera from a group of patients with SLE. Most of the patients tested by Wilcox et al were included. The blot patterns were compared with those from immunocompetent patients of similar immune response and were examined to determine compatibility with the corresponding serological results.

\section{Methods}

A total of 40 sera were tested of which 38 were from the group of patients with SLE studied by Wilcox et al. Single samples were available from 20 patients and paired sera from nine others. The time interval between the collection of paired sera ranged from nine to 36 months. Single sera were tested from two additional patients who were not part of the above series. Toxoplasma antibodies were measured by the dye test ${ }^{8}$ and by the indirect haemagglutination test (IHAT). ${ }^{9}$ Sera with a dye test titre of $>8$ or above were tested for specific IgM by $\mu$-capture enzyme linked immunosorbent assay (ELISA). ${ }^{10}$ The ELISA results were expressed in enzyme immunoassay units (EIU) with a unit value of less than 25 considered negative, $26-40$ as a doubtful positive, and above 40 as positive..$^{10}$ For comparison, control blots were prepared using three pools of sera from immunocompetent subjects. In pool 1 there was serological evidence of acute toxoplasma infection (14 sera; dye test > 16 000; IHAT 2048; IgM ELISA 226 EIU, strong positive). In pool 2 there was 
serological evidence of chronic toxoplasma infection (12 sera; dye test 128; IHAT 512; IgM ELISA 0, EIU negative). Pool 3 contained no serological evidence of toxoplasma infection (20 sera; dye test <8; IHAT <32; IgM ELISA 3 EIU, negative). The sera in the three pools were collected from specimens submitted to the Toxoplasma Reference Laboratory at Leeds. All sera were stored at $-70^{\circ} \mathrm{C}$.

\section{SDS-PAGE AND IMMUNOBLOTTING}

Electrophoresis of proteins and immunoblotting were performed as described before. ${ }^{11}$ The methods and the preparation of the toxoplasma antigen are outlined below.

Parasites were harvested from the peritoneal cavities of cotton rats inoculated three days previously with $5 \times 10^{7}$ trophozoites of the RH strain of $T$ gondii. Suitable exudates were pooled, washed, and passed through a CF11 cellulose column (Whatman, Maidstone, Kent) to remove remaining host cells. Suspensions were then subjected to three cycles of freezing and thawing ${ }^{12}$ and centrifuged. The supernatant fluid was aliquoted and stored at $-20^{\circ} \mathrm{C}$ for use as the antigen.

Sodium dodecyl sulphate polyacrylamide gel electrophoresis (SDS-PAGE) was performed using the discontinuous buffer system described by $\mathrm{Laemmli}^{13}$ with a $12 \%$ separating gel and 4\% stacking gel. Antigen samples were solubilised in SDS reducing buffer and were boiled for 6 minutes before being loaded on to the gel. Electrophoresis was performed at 200 volts until the tracking dye reached the bottom of the gel. The molecular weight standards bovine albumin, ovalbumin, glyceraldehyde-3-phosphate dehydrogenase, carbonic anhydrase, trypsinogen, soybean trypsin inhibitor and bovine lactalbumin (Sigma Chemical Co., Poole, Dorset) were also included.

Transfer of proteins to nitrocellulose paper of pore size $0.2 \mu \mathrm{m}$ (LKB, Sweden) was performed in a mini Trans-Blot cell (BioRad, California, USA) by a modification of the method of Towbin et al. ${ }^{14}$ Proteins were transferred at $100 \mathrm{~V}$ for 1 hour and the efficacy of transfer assessed by staining one strip with amido black. The other strips were soaked in 5\% non-fat dried milk in TRISsaline $(0.9 \% \mathrm{NaCl} / 10 \mathrm{mM}$ TRIS-HCl, $\mathrm{pH}$ $7 \cdot 4$ ) for 1 hour to block unused protein binding sites. After blocking the strips were washed with TRIS-saline and incubated overnight with patients' sera diluted 1 in 50 in blocking solution. The strips were then washed and incubated with horseradish peroxidase conjugated goat anti-human IgM or IgG (Sigma). After further washing the strips were developed in a solution containing 0.2 $\mathrm{mg} / \mathrm{ml}$ diaminobenzidine $/ 0 \cdot 1 \% \mathrm{H}_{2} \mathrm{O}_{2}$ in phosphate buffered saline.

\section{Results}

The serology results and blotting patterns from pool 1, the acute control (AC), and pool 2 , the chronic control (CC), are compared with those from test sera having DT titres of 8 or more in table 1. The results from pool 3, the negative control (NC), are compared with

Table 1 Summary of antibody response by serology and immunoblotting in two positive controls and in SLE patients with dye test titres of $\geqslant 8$

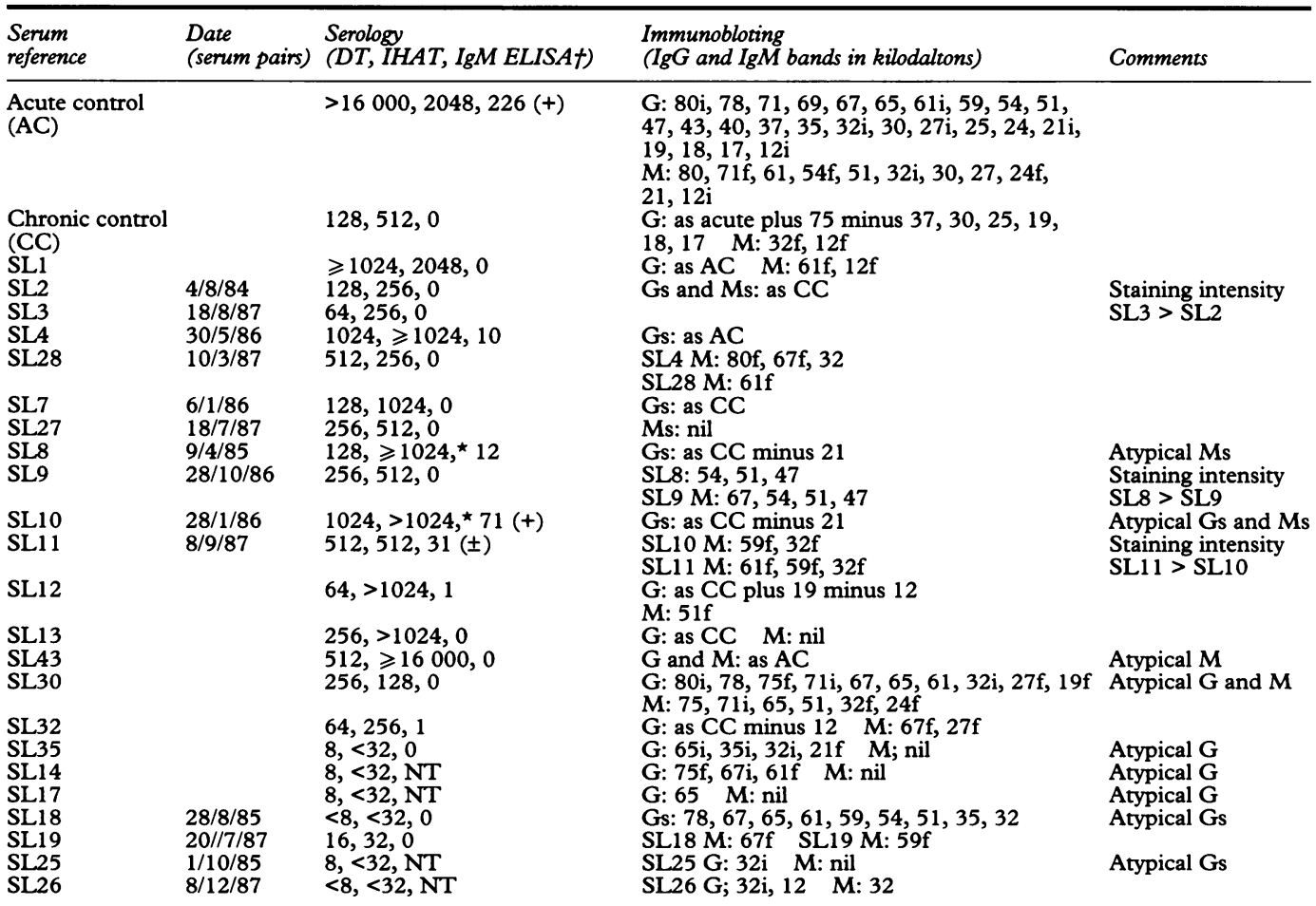

†DT = dye test; IHAT = indirect haemagglutination test: results expressed as reciprocal of titres; IgM ELISA results expressed in enzyme immunoassay units. $\mathrm{i}=$ intense, $\mathrm{f}=$ faint, $\mathrm{NT}=$ not tested. 
Table 2 Summary of antibody response by serology and immunoblotting in negative control and in SLE patients with dye test titres of $<8$

\begin{tabular}{|c|c|c|c|c|}
\hline $\begin{array}{l}\text { Serum } \\
\text { reference }\end{array}$ & $\begin{array}{l}\text { Date } \\
\text { (serum pairs) }\end{array}$ & $\begin{array}{l}\text { Serology } \\
(D T, I H A T, I g M E L I S A t)\end{array}$ & $\begin{array}{l}\text { Immunoblotting } \\
\text { (IgG and IgM bands in kilodaltons) }\end{array}$ & Comments \\
\hline $\begin{array}{l}\text { Negative control } \\
\text { (NC) }\end{array}$ & & $<8,<32,3(-)$ & $\begin{array}{l}\text { G: } 77,74,71,69,67 i, 63,59,55,50,46 i \\
42,(35,34) \text { d, } 30 f, 27 f \\
\text { M: as G plus } 32 \text { minus } 35,34,30,27\end{array}$ & \multirow{12}{*}{$\begin{array}{l}\text { Unusual intense } \\
32 \text { and } 57 \text { bands } \\
\text { Unusual intense } \\
32 \text { band }\end{array}$} \\
\hline SL5 & & $<8,32^{\star}, \mathrm{NT}$ & G: nil M: 54 & \\
\hline SL6 & & $<8,<32, \mathrm{NT}$ & G: $75,69,47 \quad M: 62$ & \\
\hline SL15 & & $<8,<32, \mathrm{NT}$ & $\mathrm{G}$ and $\mathrm{M}:$ nil & \\
\hline SL16 & & $<8,<32, \mathrm{NT}$ & G and $M$ : nil & \\
\hline SL20 & $20 / 5 / 86$ & $<8,64^{\star}, \mathrm{NT}$ & SL20 G: $66 \mathrm{f} \quad \mathrm{M}: 57 \mathrm{f}$ & \\
\hline $\begin{array}{l}\text { SL21 } \\
\text { SL22 }\end{array}$ & $21 / 8 / 87$ & $\begin{array}{l}<8,<32, \mathrm{NT} \\
<8,<32^{\star}, \mathrm{NT}\end{array}$ & $\begin{array}{l}\text { SL21 G: } 79 f,(67,66) d, 50 f, 46 f \quad M: 46 \\
\text { G: } 46 \quad \text { M: nil }\end{array}$ & \\
\hline SL23 & $4 / 7 / 84$ & $<8,<32$, NT & Gs: $57 \mathrm{f}, 32 \mathrm{i}$ & \\
\hline SL24 & $10 / 2 / 86$ & $<8,<32, \mathrm{NT}$ & Ms: $57 \mathrm{i}$ & \\
\hline SL29 & & $<8,<32, \mathrm{NT}$ & G: $72 f, 69 f, 65,61,57,51,32 f \quad M: 32 f$ & \\
\hline SL31 & & $<8,<32, \mathrm{NT}$ & $\mathrm{G}: 77 \mathrm{f}, 32 \mathrm{i} \quad \mathrm{M}: \mathrm{nil}$ & \\
\hline SL33 & & $<8,<32, \mathrm{NT}$ & G: $32 \mathrm{f} \quad \mathrm{M}: 70 \mathrm{f}, 65 \mathrm{f}, 57 \mathrm{f}, 40 \mathrm{f}$ & \\
\hline $\begin{array}{l}\text { SL34 } \\
\text { SL36 }\end{array}$ & & $\begin{array}{l}<8,<32, \text { NT } \\
<8,<32, \text { NT }\end{array}$ & $\begin{array}{ll}\text { G: } 75 f, 60 f & M: 80 f, 75 f, 70 f, 65 f, 51 f \\
\text { G: } 70 \mathrm{i}, 25 \mathrm{i} & \mathrm{M}: 78 \mathrm{f}, 63 \mathrm{f}, 55 \mathrm{f}, 32 \mathrm{f}\end{array}$ & \multirow{4}{*}{$\begin{array}{l}\text { Unusual intense } \\
70 \text { and } 25 \text { bands }\end{array}$} \\
\hline & & & & \\
\hline $\begin{array}{l}\text { SL37 } \\
\text { SL40 }\end{array}$ & & $\begin{array}{l}<8,<32, \text { NT } \\
<8,<32, \text { NT }\end{array}$ & $\begin{array}{l}\text { G: } 71 \mathrm{f}, 67 \mathrm{f}, 32 \mathrm{f} \quad \mathrm{M}: \text { nil } \\
\text { G: } 79 \mathrm{f}, 75 \mathrm{f}, 69 \mathrm{f}, 67 \mathrm{f}, 63,46 \\
\text { M: } 79 \mathrm{f}, 69 \mathrm{f}, 67 \mathrm{f}, 52 \mathrm{f}\end{array}$ & \\
\hline SLA1 & & $<8,<32, \mathrm{NT}$ & $\mathrm{G}$ and $\mathrm{M}:$ as NC & \\
\hline
\end{tabular}

†DT $=$ dye test; IHAT $=$ indirect haemagglutination test: results expressed as reciprocal of titres. $i=$ intense, $f=$ faint, $(-) \mathrm{d}=$ doublet, $\mathrm{NT}=$ not tested. ${ }^{\star}$ Non-specific agglutination.

test sera having a dye test titre of less than 8 in table 2.

IgG antibodies in the acute control serum reacted with multiple peptides, several of which were also recognised by IgM antibodies. Bands with approximate molecular weights of $80,61,32,27,21$ and 12 kilodaltons were intensely stained on the IgG blot. The 32 and 12 kilodalton bands were strongly stained on the IgM blot. The chronic control serum reacted with a smaller number of peptides on the IgG blot than the acute control. Although specific IgM was not detected in the chronic control serum, there were two faint bands on the IgM blot of 32 and 12 kilodaltons.

It is possible that the use of a $15 \%$ acrylamide separating gel would have resolved the 12 kilodalton band into smaller components. Several authors have described a very low molecular weight peptide of about 6 kilodaltons which is recognised by both IgG and IgM antibodies in acute infection ${ }^{15} 16$ but which also may be recognised in chronic infection. ${ }^{17}$

Five pairs of the test sera and six single sera had dye test titres of more than 8 and IHAT titres of more than 64 . Of these, the IgG blots from the five pairs and five of the single samples had similar profiles to either the acute control or chronic control IgG blots. Pairs SL8, 9, 10, and 11, however, showed no reaction with the 21 kilodalton peptide. SL12 and SL32 did not react with the 12 kilodalton peptide. The discrepant IgG blot from SL30 had an unusual pattern, although bands of similar molecular weight were present on one or both of the positive control IgG blots.

There was considerable variation in the IgM blots from these sera and differences were observed even between paired sera. Some of the bands on the acute IgM control were seen on the test blots-for example, those of 80 kilodaltons (SL4), 51 (SL8, 9 and 12), 54 (SL8, 9) and 61 (SL11, 28). A band of about 32 kilodaltons, which was present on both of the positive IgM controls, was seen on SL2, 3, 4, 10, 11, 30 and 43. The atypical IgM blot from SL30 bore three other bands of about 71,51 , and 24 kilodaltons which were also present on the AC IgM blot.

SL10 and 11 were the only samples that were positive by ELISA for specific IgM, suggesting a recent infection. The IgM blots were unusual, however, by comparison with those of the acute control, while the IgG blots were more typical of chronic infection. Serum SL43 also had blots which were not compatible with its serological results. The IgM blot appeared typical of acute infection but specific IgM was not detected. The IgG blot was similar to that of the acute control.

The three single sera SL35, 14, and 17 had borderline titres of 8 in the dye test and IHAT titres of less than 32. All had atypical IgG blots. The blot of SL17 was the most unusual, bearing only one band of about 65 kilodaltons. A band of the same molecular weight was present on the IgG blots from both the acute control and chronic control.

The pairs SL18, 19 and 25, 26 each had one serum with a positive dye test titre. SL19 also had a positive IHAT titre. The changes in titre may be of importance for SL18, 19, but the IgG blots were identical. The bands were surprisingly well stained in view of the low antibody titres of the sera. The peptides recognised on these IgG blots were also present on those from the acute control and chronic control. A faint band of about 67 kilodaltons was present on the SL18 IgM blot and one of 59 kilodaltons on the SL19 IgM blot. These two bands were seen on the negative control IgM blot. Of note for SL25, 26 was the intensely stained band of about 32 kilodaltons which was present on both IgG blots and the SL26 IgM blot. A band of the same molecular weight was present on the negative control IgM blot as well as the IgG and IgM blots from the acute control and chronic control. 
Despite the negative serology of the control and test sera shown in table 2, a variety of patterns were seen on both the IgG and IgM blots. No band or pattern of bands were found consistently. The molecular weights of the bands on the IgG blots ranged from about 79 to 25 kilodaltons. Certain bands, such as one of about 46 kilodaltons, were present on the IgG blots from the test sera and the negative control. Others-for example, those of 69 and 67 kilodaltons-were present on the acute control, chronic control, and negative control IgG blots.

The bands on the IgM blots ranged from about 80 to 32 kilodaltons. Once again some of these bands, such as those of 63,55 , and 46 kilodaltons, were present on the negative control IgM blot, while the 32 kilodaltons band appeared on the $\operatorname{IgM}$ blots from all three controls.

\section{Discussion}

SLE is a chronic multi-system inflammatory disorder, characterised by the production of a wide spectrum of non-organ specific autoantibodies and in particular antinuclear and DNA binding antibodies. It is now thought that the diversity of antibody reaction may be due to a quite limited number of antibodies which are polyreactive. For example, DNA binding antibodies will bind to membrane or extracellular matrix components as well as to DNA. ${ }^{18}$ Autoantibodies with a range of reactivities are a feature of certain parasitic diseses-for example, malaria. ${ }^{18}$ They may also be found in small amounts in the sera of normal subjects and so have been termed "natural" antibodies. Autoantibody production can be enhanced on exposure to exogenous antigens, as seems to be the case in malaria.

Potasman et al have demonstrated by immunoblotting that natural antibodies to $T$ gondii are present in the sera of many dye test negative adults. ${ }^{19}$ They noted that production of the antibodies seemed to be enhanced in acute toxoplasma infection, as shown by the considerable increase in staining intensity on the blots. The presence of these natural antibodies in non-immune sera would suggest that epitopes are shared between parasite antigens and those from other microorganisms or the host itself. Antigenic similarity has been shown in animals between $T$ gondii and another coccidian Hammondia hammondi, ${ }^{20}$ but this organism is not a human pathogen. Similarities between host and parasite antigens may exist because toxoplasma antibodies in apparently healthy humans have been shown to cross-react with malignant cervical tissue antigens. ${ }^{21}$

The natural antibodies described by Potasman et al have yet to be characterised, ${ }^{19}$ but it is quite possible that they include autoantibodies. As the latter tend to be polyreactive they could cross-react with parasite antigens. Alternatively, an intracellular pathogen such as $T$ gondii may incorporate on to its surface small amounts of host protein with which autoantibodies might react.
Autoantibody activity may therefore account for the reaction of non-immune sera to toxoplasma antigens. Enhanced production of autoantibodies during acute toxoplasma infection, combined with the development of specific antibodies, would then explain the increase in staining intensity on immunoblots. A high prevalence of autoantibodies has been described in some patients with neoplasia, ${ }^{22}$ and this may explain the reactivity noted by Vos. ${ }^{21}$

Wilcox et al found a significant difference in the number of dye test positive patients with SLE compared with healthy controls. ${ }^{7}$ The difference between the two groups was mostly due to a greater number of patients in the SLE group with low toxoplasma antibody titres (dye test $=8$ ). The number of patients with dye test titres of greater than 64 , however, was significantly greater in this group than in the controls. No correlation was found between toxoplasma antibody positivity and the presence of rheumatoid factors which are well known for their ability to interfere with serological assays. The raised level of toxoplasma antibodies also did not seem to be a consequence of polyclonal activation.

In our study most IgG blots from subjects with dye test and IHAT titres of more than 64 were similar to either the acute or chronic IgG control blots. The IgM blots were, however, more varied. Only sera SL10 and 11 were positive for specific IgM, suggesting a recent infection, but the $\operatorname{IgM}$ blots were not consistent with this and instead the IgG blots were more typical of chronic infection. Although the IgM ELISA for SL43 was negative, the IgM blots were typical of acute infection.

SL2 and 3 had IgM blots similar to that of the chronic control. It is not clear, however, whether the faint 32 and 12 kilodalton bands on the chronic control IgM blot represent natural antibody activity or a specific but weak response to toxoplasma peptides. A low level of specific antibody might be expected to give at least a doubtful positive reaction in the IgM ELISA unless, perhaps, there was some qualitative change in the antibody produced. The similar profile of the SL2 and 3 IgM blots and the presence of a 32 kilodalton band on the negative control IgM blot would suggest that the IgM reactivity shown by the chronic control was non-specific. In further support of this the authors of a study in which five sera from chronically infected patients were immunoblotted found that the $\operatorname{IgM}$ blots displayed only a natural antibody pattern. ${ }^{23}$

Autoantibodies present in the SLE sera might have contributed to the diversity of bands seen on the IgM blots. Certainly Potasman et al noted that the natural antibodies in their patients were usually of the IgM rather than the IgG class. ${ }^{19}$ In our study, for sera with dye test and IHAT titres of more than 64 , the bands that occurred most frequently on the $\operatorname{IgM}$ blots were of about 61 , 51 , and 32 kilodaltons. A peptide of about 32 kilodaltons has been identified in toxoplasma 
infection in humans ${ }^{15}$ and mice ${ }^{24}$ as one of the major membrane associated peptides recognised by both IgG and IgM antibodies. Because the dye test detects a small mount of IgM as well as IgG antibodies, an autoantibody of the IgM class which recognises the 32 kilodalton peptide could cross-react in the test. This would result in falsely high dye test titres for certain patients in the SLE group. Such an autoantibody might also be capable of producing false positive results in the IgM ELISA as perhaps for sera SL10 and 11.

With the exception of SL19, the remaining sera with positive dye test titres (dye test $=8$ ) had IHAT titres of less than 32. SL19 had a dye test titre of 16 and an IHAT of 32. None of these blots was compatible with chronic infection. The changes in titre between SL18 and 19 may not be significant. There were few differences between these blots, although the profiles were unusual. The results do not seem to be associated exclusively with a peptide of a particular molecular weight. A band of about 32 kilodaltons, however, was present on four of the IgG blots and on one of the IgM blots which suggests a role for crossreacting antibodies. It is therefore possible that these dye test titres could have been false positive results.

The sera with dye test values of less than 8 exhibited a variety of immunoblot patterns. There were only two cases (SL15 and 16) in which both the IgG and IgM blots had no visible bands. The bands present on the negative control and the remaining test blots were presumably due to the activity of natural antibodies as no specific antibody was detected in the dye test. Bands of similar molecular weight have been recorded in other studies of dye test negative subjects, for example those of about 32 kilodaltons ${ }^{17} 80,57,50$ and 47 kilodaltons. ${ }^{19}$

This study has shown that when attempting to diagnose toxoplasma infection in the presence of SLE, positive serological results should be interpreted with caution. Immunoblotting may prove useful in those with SLE and in other immunocompromised patients, both for confirmation of an individual's immune response and for staging infection in those who have genuine antibodies against $T$ gondii.
We thank the staff of the Toxoplasma Reference Laboratory for their help with the routine serology and Mr Trevor Woods for assistance with the photography.

1 Fleck DG. Toxoplasmosis. Public Health 1969;83:131-5.

2 Ruoss CF, Bourne GL. Toxoplasmosis in pregnancy. $f$ Obstet Gynaecol 1972;79:1115-8.

3 Wreghitt TG, Hakim M, Gray J, et al. Toxoplasmosis in heart and heart and lung transplant recipients. 7 Clin Pathol 1989;42:194-9.

4 Luft BJ, Remington JS. Toxoplasmic encephalitis. $\mathcal{F}$ Infect Dis 1988;157:1-6.

5 Ruskin J, Remington JS. Toxoplasmosis in the compromised host. Ann Intern Med 1976;84:193-9.

6 Sabin AB, Feldman HA. Dyes as microchemical indicators of a new immunity phenomenon affecting a protozoon parasite (Toxoplasma). Science 1948;108:660-3

7 Wilcox MH, Powell RJ, Pugh SF, Balfour AH. Toxoplasmosis and systemic lupus erythematosus. Ann Rheum Dis 1990;49:254-7.

8 Hunter D, Chadwick P, Balfour AH, Bridges JB. Examination of ovine foetal fluid for antibodies to Toxoplasma gondii by the dye test and an indirect Toxoplasma gondii by the dye test and an indirect 1982;138:29-33.

9 Hunter D, Chadwick P, Balfour AH, Bridges JB. An assessment of a commercially available haemagglutination test for detecting Toxoplasma antibodies in ovine sera. Br Vet ₹ 1980;136:339-42

10 Payne RA, Joynson DHM, Balfour AH, et al. Public Health Laboratory Service enzyme linked immunosorbent assay for detecting Toxoplasma specific IgM antibody. $₹$ Clin Pathol 1987;40:276-81.

11 Noel I, Balfour AH. Immunoblot analysis of IgM and IgG subclass responses in murine toxoplasmosis. IgG subclass responses in murine toxoplasm

12 Francis JM. A contribution towards the antigenic analysis of Toxoplasma gondii. Med Lab Sci 1983;40:319-25.

13 Laemmli UK. Cleavage of structural proteins during the assembly of the head of bacteriophage T4. Nature 1970;227:680-5

14 Towbin H, Staehelin T, Gordon J. Electrophoretic transfer of proteins from polyacrylamide gels to nitrocellulose sheets: Procedure and some applications. Proc Natl Acad Sci USA 1979;76:4350-4.

15 Sharma SD, Mullenax J, Araujo FG, Erlich HA, Remington JS. Western blot analysis of the antigens of Toxoplasma gondii recognised by human IgM and IgG antibodies. F Immunol 1983;131:977-83.

16 Partanen P, Turunen HJ, Paasivuo RT, Leinikki PO. Immunoblot analysis of Toxoplasma gondii antigens by human immunoglobulins $\mathrm{G}, \mathrm{M}$, and $\mathrm{A}$ antibodies at human immunoglobulins $G, M$, and A antibodies at different

17 Moir IL, Davidson MM, Ho-Yen DO. Comparison of IgG antibody profiles by immunoblotting in patients with acute and previous Toxoplasma gondii infection. $\mathcal{F}$ Clin Pathol 1991;44:569-72.

18 Wozencraft AO, Staines NA. DNA-binding antibodies and parasitic diseases. Parasitol Today 1990;6:254-9.

19 Potasman I, Araujo FG, Remington JS. Toxoplasma antigens recognised by naturally occurring human antibodies. I Clin Microbiol 1986;24:1050-4.

20 Araujo FG, Dubey JP, Remington JS. Antigenic similarity between the coccidian parasites Toxoplasma gondii and between the coccidian parasites Toxoplasma gondii and

Hammondia hammondi. $\mathcal{F}$ Protozool $1984 ; 31: 145-7$.
21 Vos GH. Population studies showing cross-reactivity of Toxoplasma gondii antibodies with antibodies to malignant cervical tissue antigens. South African Med $\mathcal{F}$ 1987;71:78-82.

22 Fernandez-Madrid F, Mattioli M. Antinuclear antibodies (ANA): Immunologic and clinical significance. Semin Arthritis Rheum 1976;6:83-124.

23 Potasman I, Araujo FG, Desmonts G, Remington JS Analysis of Toxoplasma gondii antigens recognised by human sera obtained before and after acute infection. Infect Dis 1986;154:650-7.

24 Kasper LH. Identification of stage-specific antigens of Toxoplasma gondii. Infect Immun 1989;57:668-72. 